\title{
Properties of perpetual integral functionals of Brownian motion with drift
}

\author{
Paavo Salminen ${ }^{\mathrm{a}, *}$, Marc Yor ${ }^{\mathrm{b}}$ \\ a Åbo Akademi, Mathematical Department, 20500 Åbo, Finland \\ ${ }^{\mathrm{b}}$ Université Pierre et Marie Curie, laboratoire de probabilités, 4, place Jussieu, case 188, 75252 Paris cedex 05, France
}

Received 17 September 2003; accepted 13 January 2004

Available online 31 March 2005

\section{Abstract}

In this paper we study integrability properties of the random variable

$$
I_{\infty}(f):=\int_{0}^{\infty} f\left(B_{t}^{(\mu)}\right) \mathrm{d} t,
$$

where $\left\{B_{t}^{(\mu)}: t \geqslant 0\right\}$ is a Brownian motion with drift $\mu>0$ and $f$ is a non-negative, Borel measurable function. In particular, we find conditions under which $I_{\infty}(f)$ (i) is finite a.s., (ii) has all the moments, (iii) has some exponential moments, and (iv) has bounded potential.

() 2005 Elsevier SAS. All rights reserved.

\section{Résumé}

Dans ce travail, nous étudions les propriétés d'intégrabilité de la variable aléatoire :

$$
I_{\infty}(f):=\int_{0}^{\infty} f\left(B_{t}^{(\mu)}\right) \mathrm{d} t
$$

où $\left\{B_{t}^{(\mu)}: t \geqslant 0\right\}$ est un mouvement brownien avec dérive $\mu>0$, et $f$ une fonction borélienne positive. En particulier, nous trouvons des conditions pour lesquelles $I_{\infty}(f)$ (i) est finie p.s.; (ii) possède des moments de tous ordres; (iii) possède des moments exponentiels; (iv) a un potentiel borné.

() 2005 Elsevier SAS. All rights reserved.

MSC: 60J65; 60J60; 60J70

Keywords: Local time; Green function; Kac's moment formula; Khas'minskii's lemma; Last exit time

\footnotetext{
* Corresponding author.

E-mail address: phsalmin@abo.fi (P. Salminen).
} 


\section{Introduction}

Let $B^{(\mu)}=\left\{B_{t}^{(\mu)}:=B_{t}+\mu t: t \geqslant 0\right\}$ be a Brownian motion with drift $\mu>0, \operatorname{BM}(\mu)$, for short, and $f$ a non-negative measurable function. In this paper we study integrability properties of the functionals of the type

$$
I_{\infty}(f):=\int_{0}^{\infty} f\left(B_{s}^{(\mu)}\right) \mathrm{d} s .
$$

We take $\mu>0$ only to simplify the presentation. Corresponding properties of functionals for a Brownian motion with negative drift are easily deduced from the case with positive drift.

The above kind of functionals appear in many applications. We have in mind especially financial mathematical framework where $I_{\infty}(f)$ is interpreted as a continuous perpetuity. We refer to Dufresne [7] where it is seen how the functional

$$
\int_{0}^{\infty} \exp \left(-2 B_{s}^{(\mu)}\right) \mathrm{d} s
$$

arises as a perpetuity after a limiting procedure in a discrete model.

It is of great importance to have clear and easy-to-apply criteria in terms of $f$ under which $I_{\infty}(f)$

(i) is finite a.s.,

(ii) has all the moments,

(iii) has some exponential moments,

(iv) has a bounded potential.

In some cases, it is possible to compute explicitly the distribution or the Laplace transform of $I_{\infty}(f)$ and from this, of course, to check the validity of (i)-(iv). For instance, we have (see Dufresne [7])

$$
\int_{0}^{\infty} \exp \left(-2 B_{s}^{(\mu)}\right) \mathrm{d} s \stackrel{(\mathrm{d})}{=} \frac{1}{2 \gamma_{\mu}},
$$

where $\gamma_{\mu}$ is a gamma-distributed random variable with parameter $\mu$ and $\stackrel{(\mathrm{d})}{=}$ reads "is identical in law with". Consequently, Dufresne's functional (1) has finite $m$ th moment if and only if $m<\mu$. We remark also that in Yor [29] (see [30] for an English translation) it is shown that

$$
\int_{0}^{\infty} \exp \left(-2 B_{s}^{(\mu)}\right) \mathrm{d} s \stackrel{(\mathrm{d})}{=} \inf \left\{t: R_{t}^{(\delta)}=0\right\},
$$

where $R^{(\delta)}$ is a Bessel process of dimension $\delta=2(1-\mu)$ started at 1 . One motivation for the present paper was, indeed, the desire to find "natural" functionals which could serve as a perpetuity having some exponential moments. Our criteria show that a suitable candidate is the functional

$$
\int_{0}^{\infty}\left(1+a \exp \left(B_{s}^{(\mu)}\right)\right)^{-2} \mathrm{~d} s, \quad a>0 .
$$

We noticed, independently of the present study, that the Laplace transform of this functional can be computed explicitly in the case $\mu=1 / 2$. However, we postpone to a forthcoming paper [24] the detailed discussions of the different methods of computing the distributions of perpetual functionals using, in particular, some connections 
between perpetual functionals and first hitting times, while, in the present paper, we address only the question of finding criteria for (i) to (iv).

We now shortly review the literature known to us on the questions (i)-(iv).

Firstly, in Engelbert and Senf [13] a necessary and sufficient condition for (i) to hold is found. The result is proved in [13] by formulating the problem for ordinary Brownian motion and applying Shepp's dichotomy theorem (see Shepp [25]). Our proof, presented in Section 3, is based on Jeulin's lemma [16] and is, perhaps, more straightforward in the sense that we work directly with $B^{(\mu)}$ and its local time.

Secondly, we remark that there has been much interest focused on finding conditions for a.s. finiteness of integral functionals of Bessel processes (see, e.g., Engelbert and Schmidt [10], Pitman and Yor [22,23], Engelbert and Schmidt [12], Xue [28], Csörgö, Horváth and Shao [5], and Cherny [3]). In particular, Jeulin's lemma is applied in [22] and [23].

Thirdly, recall that if

$$
\sup _{x} \mathbf{E}_{x}\left(I_{\infty}(f)\right)<\infty
$$

that is, $I_{\infty}(f)$ has a bounded potential, then $I_{\infty}(f)$ has some exponential moments. This result is usually called Khas'minskii's lemma (see Simon [26], Durrett [8], and Chung and Zhao [4]). For a recent discussion and generalizations of Khas'minskii's lemma, see Stummer and Sturm [27]. Our approach is, however, based on a more general result (see Lemma 5.2) presented in Dellacherie and Meyer [6], Chapitre VI, Théorème 105, p. 108.

Finally, we are not aware of any works where conditions in terms of $f$ for (ii), (iii) and (iv) are discussed. However, for exponential moments of integral functionals of Brownian motion over a finite time interval, see Engelbert and Schmidt [11] whose result is shortly explained in Remark 5.3(4) below.

The paper is organised so that in the next section we introduce some notation, summarize our results in a table form, and make some further comments. The proofs of the criteria for (i), (ii), (iii) and (iv) are then given in the Sections $3,4,6$, and 5 , respectively.

\section{Notation and summary of the results}

It is assumed throughout the paper that $\mu>0$ and the function $f$ appearing in the functional

$$
I_{\infty}(f):=\int_{0}^{\infty} f\left(B_{s}^{(\mu)}\right) \mathrm{d} s,
$$

satisfies the condition

(A) $f$ is a non-negative and locally integrable function.

The main results are summarized in Table 1. The classes indicated in the table consist of the functions $f$ satisfying (A) and for which the condition in the first column holds. For instance, $f$ is in $\mathcal{K}_{4}$ if it is non-negative and locally integrable and has bounded potential, i.e.,

$$
\sup _{x} \mathbf{E}_{x}\left(I_{\infty}(f)\right)<\infty .
$$

Because of the criterion for $\mathcal{K}_{1}$, a necessary condition for a function $f$ to belong to $\mathcal{K}_{i}, i=2,3,4$, is that it is integrable at $+\infty$ :

$$
\int^{\infty} f(x) \mathrm{d} x<\infty .
$$


Table 1

\begin{tabular}{|c|c|c|}
\hline Class & & Criterion \\
\hline$\overline{\mathcal{K}_{1}: I_{\infty}(f)<\infty \text { a.s. }}$ & $\Leftrightarrow$ & $\int^{\infty} f(x) \mathrm{d} x<\infty$ \\
\hline $\mathcal{K}_{2}: \forall n \in \mathbf{N}, \forall x \quad \mathbf{E}_{x}\left(I_{\infty}(f)^{n}\right)<\infty$ & $\Leftarrow$ & $\begin{array}{l}\int_{\exists}^{\infty} f(x) \mathrm{d} x<\infty \quad \text { and } \\
\exists m>0 \quad f(x)=\mathrm{O}\left(|x|^{m}\right)_{x \rightarrow-\infty}\end{array}$ \\
\hline $\mathcal{K}_{3}: \exists \gamma>0, \forall x \quad \mathbf{E}_{x}\left(\exp \left(\gamma I_{\infty}(f)\right)\right)<\infty$ & $\Leftarrow$ & $\begin{array}{l}\int^{\infty} f(x) \mathrm{d} x<\infty \quad \text { and } \\
f(x)=\mathrm{O}(1)_{x \rightarrow-\infty}\end{array}$ \\
\hline $\mathcal{K}_{4}: \sup _{x} \mathbf{E}_{x}\left(I_{\infty}(f)\right)<\infty$ & $\Leftrightarrow$ & $\int_{-\infty}^{\infty} f(x) \mathrm{d} x<\infty$ \\
\hline
\end{tabular}

Thus, as seen from the table, for $f$ to belong to $\mathcal{K}_{i}, i=2,3,4$, only its behaviour at $-\infty$ is of interest.

It is clear from the definitions that

$$
\mathcal{K}_{3} \subset \mathcal{K}_{2} \subset \mathcal{K}_{1} \text {. }
$$

Notice from the table that $\mathcal{K}_{4}$ contains functions for which the sufficient criterion for $\mathcal{K}_{3}$ (or for $\mathcal{K}_{2}$ ) does not hold. However, we still have

$$
\mathcal{K}_{4} \subset \mathcal{K}_{3}
$$

This fact is a special case of a more general result, often called Khas'minskii's lemma (see Lemma 5.2 below and discussion therein).

Moreover, the table shows that $\mathcal{K}_{1}$ and $\mathcal{K}_{4}$ do not depend on the particular value of the positive drift parameter $\mu$. In fact, letting $\mathcal{L}_{i}^{(\mu)}, i=1,2,3,4$, be the family of the distributions of the functionals

$$
\int_{0}^{\infty} f\left(B_{s}^{(\mu)}\right) \mathrm{d} s, \quad f \in \mathcal{K}_{i}, i=1,2,3,4,
$$

respectively, we have the following

Proposition 2.1. The families $\mathcal{L}_{i}^{(\mu)}, i=1,2,3,4$, do not depend on $\mu>0$.

Proof. By the scaling property of Brownian motion we have for any $c>0$

$$
\int_{0}^{\infty} f\left(B_{s}+\mu s\right) \mathrm{d} s \stackrel{(\mathrm{d})}{=} c^{2} \int_{0}^{\infty} f\left(c B_{s}+\mu c^{2} s\right) \mathrm{d} s .
$$

Choosing here $c=1 / \mu$ gives

$$
\int_{0}^{\infty} f\left(B_{s}+\mu s\right) \mathrm{d} s \stackrel{(\mathrm{d})}{=} \frac{1}{\mu^{2}} \int_{0}^{\infty} f\left(\frac{1}{\mu}\left(B_{s}+s\right)\right) \mathrm{d} s .
$$

but this means that $\mathcal{L}_{i}^{(\mu)}=\mathcal{L}_{i}^{(1)}, i=1,2,3,4$, proving the claim.

\section{Finiteness - class $\mathcal{K}_{1}$}

We now prove the characterization of $\mathcal{K}_{1}$ given in the first row of the table above. As stated in the Introduction, the result can be found in Engelbert and Senf [13]. Their proof is based on Shepp's dichotomy theorem (see 
Shepp [25]). Our proof is different and, perhaps, simpler, and we think that it is worthwhile - also to make the paper more readable and self-contained - to present it. An important tool in our proof is the following lemma due to Jeulin (see [16], p. 254, Proposition 4, or [15]) formulated here in a slightly less general form. We also refer to Pitman and Yor [22] and [23] for applications of Jeulin's lemma for integral functionals of Bessel processes.

Lemma 3.1. Let $\left\{Z_{y}: y \geqslant 0\right\}$ be a stochastic process such that the law of $Z_{y}$ does not depend on $y$ and is absolutely continuous with respect to the Lebesgue measure. Assume also that $\mathbf{E}\left|Z_{1}\right|<\infty$. Then for any $\sigma$-finite measure $v$ on $\mathbf{R}_{+}(:=$the set of non-negative real numbers)

$$
\mathbf{P}\left(\int_{0}^{\infty}\left|Z_{y}\right| v(\mathrm{~d} y)<\infty\right)=1 \Leftrightarrow v\left(\mathbf{R}_{+}\right)<\infty .
$$

The proof of Proposition 3.3 below is based on the properties of local time of Brownian motion with drift. The Ray-Knight theorems form a rich source of information for the local time processes. Although we here only need a small fragment of this information, it is perhaps good to recall the complete statement (taken from [2], V. 11, p. 90) of the Ray-Knight theorem (formulated here as a lemma) for the total local time process of Brownian motion with drift. We let $L^{(\mu)}(t, y)$ denote the local time of $B^{(\mu)}$ at level $y$ and at time $t$ (taken with respect to the Lebesgue measure). The total local time is defined via

$$
L^{(\mu)}(\infty, y):=\lim _{t \rightarrow \infty} L^{(\mu)}(t, y) .
$$

Lemma 3.2. Conditionally on $\inf \left\{B_{s}^{(\mu)}: s \geqslant 0\right\}=m$ and $B_{0}^{(\mu)}=0$ it holds

$$
\left\{L^{(\mu)}(\infty, m+y): 0 \leqslant y \leqslant-m\right\} \stackrel{(\mathrm{d})}{=}\left\{X_{y}^{(4)}: 0 \leqslant y \leqslant-m\right\},
$$

and

$$
\left\{L^{(\mu)}(\infty, y): y \geqslant 0\right\} \stackrel{(\mathrm{d})}{=}\left\{X_{y}^{(2)}: y \geqslant 0\right\},
$$

where $X^{(4)}$ is a diffusion with the generator

$$
2 z \frac{\mathrm{d}^{2}}{\mathrm{~d} z^{2}}+(4-2 \mu z) \frac{\mathrm{d}}{\mathrm{d} z}
$$

and $X^{(2)}$ is a diffusion with the generator

$$
2 z \frac{\mathrm{d}^{2}}{\mathrm{~d} z^{2}}+(2-2 \mu z) \frac{\mathrm{d}}{\mathrm{d} z}
$$

The process $X^{(2)}$ is started from the location of $X^{(4)}$ at time $-m$, but otherwise $X^{(4)}$ and $X^{(2)}$ are independent.

We now apply Lemmas 3.1 and 3.2 to characterize the class $\mathcal{K}_{1}$.

Proposition 3.3. $\left(\mathcal{K}_{1}\right)$ Assume that the function $f$ satisfies the assumption (A). Then

$$
\int_{0}^{\infty} f\left(B_{t}^{(\mu)}\right) \mathrm{d} t<\infty \text { a.s. } \Leftrightarrow \int^{\infty} f(y) \mathrm{d} y<\infty .
$$


Proof. The starting point is the occupation time formula for $\operatorname{BM}(\mu)$

$$
\int_{0}^{t} f\left(B_{s}^{(\mu)}\right) \mathrm{d} s=\int_{\mathbf{R}} f(y) L^{(\mu)}(t, y) \mathrm{d} y .
$$

Letting here $t \rightarrow \infty$ we obtain by monotone convergence

$$
\int_{0}^{\infty} f\left(B_{s}^{(\mu)}\right) \mathrm{d} s=\int_{\mathbf{R}} f(y) L^{(\mu)}(\infty, y) \mathrm{d} y
$$

In view of (3) we wish to use Lemma 3.1 with $Z_{y}=L^{(\mu)}(\infty, y)$ and $v(\mathrm{~d} y)=f(y) \mathrm{d} y$. Firstly, because $f$ is assumed to be locally integrable and non-negative, it is clear that $v$ is a $\sigma$-finite measure. Secondly, it follows from Lemma 3.2 (see also [2], p. 90 and 2.1.3.4(2), p. 253)

$$
\mathbf{P}_{x}\left(L^{(\mu)}(\infty, y) \geqslant v\right)= \begin{cases}\mathrm{e}^{-\mu v}, & y \geqslant x, \\ \mathrm{e}^{-2 \mu(x-y)} \mathrm{e}^{-\mu v}, & y \leqslant x .\end{cases}
$$

In particular, the $\mathbf{P}_{0}$-distribution of $L^{(\mu)}(\infty, y)$ for $y \geqslant 0$ is independent of $y$. Setting

$$
\hat{f}(x):= \begin{cases}f(x), & x \geqslant 0 \\ 0, & x<0\end{cases}
$$

we obtain

$$
\mathbf{P}_{0}\left(\int_{0}^{\infty} \hat{f}\left(B_{s}^{(\mu)}\right) \mathrm{d} s<\infty\right)=1 \Leftrightarrow \int_{0}^{\infty} \hat{f}(x) \mathrm{d} x<\infty \Leftrightarrow \int_{0}^{\infty} f(x) \mathrm{d} x<\infty .
$$

Notice next that

$$
\int_{0}^{\infty} f\left(B_{s}^{(\mu)}\right) \mathrm{d} s=\int_{0}^{\lambda_{0}} f\left(B_{s}^{(\mu)}\right) \mathrm{d} s+\int_{\lambda_{0}}^{\infty} f\left(B_{s}^{(\mu)}\right) \mathrm{d} s
$$

where $\lambda_{0}:=\sup \left\{t: B_{t}^{(\mu)}=0\right\}$. Because $\lambda_{0}<\infty$ a.s., the first integral on the right-hand side of (5) is a.s. finite. For the second integral we have (because $f \geqslant 0$ )

$$
\int_{\lambda_{0}}^{\infty} f\left(B_{s}^{(\mu)}\right) \mathrm{d} s \leqslant \int_{0}^{\infty} \hat{f}\left(B_{s}^{(\mu)}\right) \mathrm{d} s .
$$

On the other hand,

$$
\int_{0}^{\infty} \hat{f}\left(B_{s}^{(\mu)}\right) \mathrm{d} s \leqslant \int_{0}^{\infty} f\left(B_{s}^{(\mu)}\right) \mathrm{d} s,
$$

and this completes the proof.

Remark 3.4. Let $v$ be a measure on $\mathbf{R}$, which is finite on compacts, and define

$$
I_{\infty}(\nu):=\int_{\mathbf{R}} v(\mathrm{~d} y) L^{(\mu)}(\infty, y) .
$$


Then it can be proved similarly as above that

$$
I_{\infty}(v)<\infty \text { a.s. } \Leftrightarrow \int^{\infty} v(\mathrm{~d} y)<\infty .
$$

\section{Moments - class $\mathcal{K}_{2}$}

Proposition 4.1. $\left(\mathcal{K}_{2}\right)$ Assume that the function $f$ satisfies $(\mathrm{A})$, is integrable at $+\infty$, and for some positive integer $m$

$$
f(x)=\mathrm{O}\left(|x|^{m}\right)_{x \rightarrow-\infty} .
$$

Then for all $x$ and $n=1,2, \ldots$

$$
v^{(n)}(x):=\mathbf{E}_{x}\left(I_{\infty}(f)^{n}\right)<\infty .
$$

Proof. We first compute $v^{(1)}$, the mean of $I_{\infty}(f)$. Let $p^{(\mu)}$ be the transition density of $\operatorname{BM}(\mu)$ such that

$$
\mathbf{P}_{x}\left(B_{t}^{(\mu)} \in \mathrm{d} y\right)=p^{(\mu)}(t ; x, y) m(\mathrm{~d} y),
$$

where $m(\mathrm{~d} y)=2 \exp (2 \mu y) \mathrm{d} y$ is the speed measure of $\mathrm{BM}(\mu)$. As is well known, the Green kernel is given by

$$
G_{0}(x, y):=\int_{0}^{\infty} p^{(\mu)}(t ; x, y) \mathrm{d} t=\frac{1}{2 \mu} \mathrm{e}^{-2 \mu x} \quad \text { for } x \geqslant y,
$$

and this determines the value of the integral also for $x \leqslant y$ due to the fact that $p^{(\mu)}(t ; x, y)=p^{(\mu)}(t ; y, x)$. Consider now

$$
\begin{aligned}
v^{(1)}(x) & =\mathbf{E}_{x}\left(\int_{0}^{\infty} f\left(B_{t}^{(\mu)}\right) \mathrm{d} t\right)=\int_{0}^{\infty} \mathbf{E}_{x}\left(f\left(B_{t}^{(\mu)}\right)\right) \mathrm{d} t=\int_{0}^{\infty} \mathrm{d} t \int_{\mathbf{R}} m(\mathrm{~d} y) p^{(\mu)}(t ; x, y) f(y) \\
& =\int_{\mathbf{R}} m(d y) f(y) \int_{0}^{\infty} \mathrm{d} t p^{(\mu)}(t ; x, y),
\end{aligned}
$$

and so, from (7),

$$
v^{(1)}(x)=\mathbf{E}_{x}\left(I_{\infty}(f)\right)=\frac{1}{\mu} \int_{x}^{+\infty} f(y) \mathrm{d} y+\frac{1}{\mu} \mathrm{e}^{-2 \mu x} \int_{-\infty}^{x} f(y) \mathrm{e}^{2 \mu y} \mathrm{~d} y .
$$

Due to (6) and the integrability of $f$ at $+\infty$ it is clear that $v^{(1)}(x)$ is finite for all $x$. Further, for any real number $N$

$$
\begin{aligned}
\limsup _{x \rightarrow+\infty} v^{(1)}(x) & =\limsup _{x \rightarrow+\infty} \frac{1}{\mu} \mathrm{e}^{-2 \mu x} \int_{-\infty}^{x} f(y) \mathrm{e}^{2 \mu y} \mathrm{~d} y=\limsup _{x \rightarrow+\infty} \frac{1}{\mu} \mathrm{e}^{-2 \mu x} \int_{N}^{x} f(y) \mathrm{e}^{2 \mu y} \mathrm{~d} y \\
& \leqslant \frac{1}{\mu} \int_{N}^{\infty} f(y) \mathrm{d} y
\end{aligned}
$$


which gives

$$
\lim _{x \rightarrow+\infty} v^{(1)}(x)=0
$$

Because $f$ is locally integrable and satisfies (6) it follows from (8) that

$$
v^{(1)}(x)=\mathrm{O}\left(|x|^{m+1}\right)_{x \rightarrow-\infty} .
$$

Next recall the Kac moment formula (see Fitzsimmons and Pitman [14], or [2], II.27, p. 31)

$$
v^{(n)}(x)=n \int_{\mathbf{R}} G_{0}(x, y) v^{(n-1)}(y) f(y) m(\mathrm{~d} y),
$$

or, equivalently,

$$
v^{(n)}(x)=\frac{n}{\mu} \int_{x}^{+\infty} f(y) v^{(n-1)}(y) \mathrm{d} y+\frac{n}{\mu} \mathrm{e}^{-2 \mu x} \int_{-\infty}^{x} f(y) v^{(n-1)}(y) \mathrm{e}^{2 \mu y} \mathrm{~d} y .
$$

Take here $n=2$ and use (9) and (10) to show that $v^{(2)}(x)$ is finite. Proceeding inductively, it is seen that

$$
\lim _{x \rightarrow+\infty} v^{(n)}(x)=0 \quad \text { and } \quad v^{(n)}(x)=\mathrm{O}\left(|x|^{m+n}\right)_{x \rightarrow-\infty},
$$

which implies, from (11), the existence of $v^{(n+1)}$ and, further, (13) holds by a recurrence argument, thus completing the proof.

Next examples show that if the polynomial growth condition (6) is not valid then the functional does not, in general, have all the moments.

Example 4.2. (1) For $f(x)=\exp (-\mu x)$ we have (cf. (8))

$$
\mathbf{E}_{x}\left(\int_{0}^{\infty} \exp \left(-\mu B_{t}^{(\mu)}\right) \mathrm{d} t\right)=\frac{2}{\mu^{2}} \exp (-\mu x)
$$

and from (11) (or (12)) with $n=2$

$$
\mathbf{E}_{x}\left(\left(\int_{0}^{\infty} \exp \left(-\mu B_{t}^{(\mu)}\right) \mathrm{d} t\right)^{2}\right)=+\infty
$$

(2) Dufresne's functional (1), i.e., $f(x)=\exp (-2 x)$, is also easy to analyze via (12). In particular, it is seen without knowing the explicit law - that this functional has a finite $m$ th moment if and only if $m<\mu$.

\section{Bounded potential - class $\mathcal{K}_{4}$}

We discuss the class $\mathcal{K}_{4}$ before the class $\mathcal{K}_{3}$ to be able to use these results when analysing $\mathcal{K}_{3}$.

Proposition 5.1. $\left(\mathcal{K}_{4}\right)\left(\right.$ i) The integral functional $I_{\infty}(f)$ has bounded potential, that is,

$$
p_{o}:=\sup _{x} \mathbf{E}_{x}\left(I_{\infty}(f)\right)<\infty,
$$


if and only if

$$
\int_{-\infty}^{\infty} f(y) \mathrm{d} y<\infty .
$$

In this case,

$$
p_{o}=\frac{1}{\mu} \int_{-\infty}^{\infty} f(y) \mathrm{d} y .
$$

(ii) $I_{\infty}(f)$ has exponential moments for $\gamma<1 / p_{o}$, i.e., for all $x$

$$
\mathbf{E}_{x}\left(\exp \left(\gamma I_{\infty}(f)\right)\right)<\infty .
$$

More precisely, for all $x$ and $\varepsilon \in(0,1)$

$$
\mathbf{E}_{x}\left(\exp \left(\frac{1-\varepsilon}{p_{o}} I_{\infty}(f)\right)\right) \leqslant \varepsilon^{-1}
$$

The statement that (14) implies (15) can be proved directly using the Kac moment formula. We remark also that it is a special case of Khas'minskii's well-known lemma (see Khas'minskii [19], Simon [26], Durrett [8], and Chung and Zhao [4]). For a more recent discussion and generalizations of Khas'minskii's lemma, see Stummer and Sturm [27]. However, we want to point out here that this statement (as well as Khas'minskii's lemma) can be deduced from the following more general result presented in Dellacherie and Meyer [6], Chapitre VI, Théorème 105, p. 108.

Lemma 5.2. Let $A$ be a continuous, $\left(\mathcal{F}_{t}\right)$-adapted, non-decreasing process starting at 0 such that there exists a constant $C>0$ satisfying

$$
\forall t \geqslant 0 \quad \mathbf{E}\left(A_{\infty}-A_{t} \mid \mathcal{F}_{t}\right) \leqslant C .
$$

Then

(i) $\forall n \in \mathbf{N}, t \geqslant 0, \mathbf{E}\left(\left(A_{\infty}-A_{t}\right)^{n} \mid \mathcal{F}_{t}\right) \leqslant n ! C^{n}$,

(ii) $\forall n \in \mathbf{N}, \mathbf{E}\left(A_{\infty}^{n}\right) \leqslant n ! C^{n}$,

(iii) $\mathbf{E}\left(\exp \left(\lambda A_{\infty}\right)\right) \leqslant 1 /(1-\lambda C)$ for $\lambda<1 / C$, and, consequently,

$$
\mathbf{E}\left(\exp \left(\frac{1-\varepsilon}{C} A_{\infty}\right)\right) \leqslant \varepsilon^{-1}
$$

for $\varepsilon \in(0,1)$.

Proof. Clearly, (ii) follows from (i) by taking $t=0$, and (iii) from (ii) using the series expansion of the exponential function. To prove (i) consider the case $n=2$

$$
\begin{aligned}
\mathbf{E}\left(\left(A_{\infty}-A_{t}\right)^{2} \mid \mathcal{F}_{t}\right) & =\mathbf{E}\left(\left(\int_{t}^{\infty} \mathrm{d} A_{s}\right)^{2} \mid \mathcal{F}_{t}\right)=2 ! \mathbf{E}\left(\int_{t}^{\infty} \mathrm{d} A_{s_{1}} \int_{s_{1}}^{\infty} \mathrm{d} A_{s_{2}} \mid \mathcal{F}_{t}\right) \\
& =2 \mathbf{E}\left(\int_{t}^{\infty} \mathrm{d} A_{s_{1}}\left(A_{\infty}-A_{s_{1}}\right) \mid \mathcal{F}_{t}\right)=2 \mathbf{E}\left(\int_{t}^{\infty} \mathrm{d} A_{s_{1}} \mathbf{E}\left(A_{\infty}-A_{s_{1}} \mid \mathcal{F}_{s_{1}}\right) \mid \mathcal{F}_{t}\right) \leqslant 2 C^{2},
\end{aligned}
$$


where the next to the last step is obtained by replacing the process $\left\{A_{\infty}-A_{u}: u \geqslant 0\right\}$ by its optional projection $\left\{\mathbf{E}\left(A_{\infty}-A_{u} \mid \mathcal{F}_{u}\right): u \geqslant 0\right\}$. The general case is only notationally more complicated, and we skip the details.

Proof of Proposition 5.1. To compute the supremum in (14), consider (cf. (8))

$$
\mathbf{E}_{x}\left(I_{\infty}(f)\right)=\frac{1}{\mu} \int_{x}^{+\infty} f(y) \mathrm{d} y+\frac{1}{\mu} \mathrm{e}^{-2 \mu x} \int_{-\infty}^{x} f(y) \mathrm{e}^{2 \mu y} \mathrm{~d} y \geqslant \frac{1}{\mu} \int_{x}^{+\infty} f(y) \mathrm{d} y .
$$

Hence,

$$
\sup _{x}\left\{\mathbf{E}_{x}\left(I_{\infty}(f)\right)\right\} \geqslant \frac{1}{\mu} \int_{-\infty}^{\infty} f(y) \mathrm{d} y .
$$

From (18) it also follows that

$$
\mathbf{E}_{x}\left(I_{\infty}(f)\right) \leqslant \frac{1}{\mu} \int_{-\infty}^{\infty} f(y) \mathrm{d} y
$$

and so, as claimed,

$$
p_{o}:=\sup _{x}\left\{\mathbf{E}_{x}\left(I_{\infty}(f)\right)\right\}=\frac{1}{\mu} \int_{-\infty}^{+\infty} f(y) \mathrm{d} y .
$$

Take now in Lemma 5.2

$$
A_{t}:=\int_{0}^{t} f\left(B_{s}^{(\mu)}\right) \mathrm{d} s
$$

Then (17) holds with $C=p_{o}$, and from (iii) in Lemma 5.2 we obtain (16).

Remark 5.3. (1) As in Remark 3.4, consider the functional

$$
I_{\infty}(v):=\int_{\mathbf{R}} v(\mathrm{~d} y) L^{(\mu)}(\infty, y) .
$$

Then it can be proved similarly as above that

$$
p_{o}(v):=\sup _{x} \mathbf{E}_{x}\left(I_{\infty}(v)\right)=\frac{1}{\mu} v(\mathbf{R})
$$

Notice that if we take $\nu(\mathrm{d} x)=\varepsilon_{y}(\mathrm{~d} x)$, Dirac's measure at $y>0$, then $p_{o}=1 / \mu$. Therefore, from (15), for $\gamma<\mu$

$$
\mathbf{E}_{0}\left(\exp \left(\gamma L^{(\mu)}(\infty, y)\right)\right)<\infty
$$

which is the best possible bound since

$$
\mathbf{P}_{0}\left(L^{(\mu)}(\infty, y)\right) \in \mathrm{d} l=\mu \mathrm{e}^{-\mu l} \mathrm{~d} l .
$$

(2) Let $\sigma=\left\{\sigma_{t}: t \geqslant 0\right\}, \sigma_{0}=0$, be a subordinator and $h:[0, \infty) \mapsto[0, \infty)$ a decreasing function. In Bertoin [1], p. 28, it is proved that the following three statements are equivalent 
(i) $\mathbf{E}\left(\int_{0}^{\infty} h\left(\sigma_{t}\right) \mathrm{d} t\right)<\infty$,

(ii) $\mathbf{P}\left(\int_{0}^{\infty} h\left(\sigma_{t}\right) \mathrm{d} t<\infty\right)=1$,

(iii) $\mathbf{P}\left(\int_{0}^{\infty} h\left(\sigma_{t}\right) \mathrm{d} t<\infty\right)>0$.

From Lemma 5.2 it follows that (i) (and hence also (ii) and (iii)) are equivalent with

(iv) $\mathbf{E}\left(\exp \left(\gamma \int_{0}^{\infty} h\left(\sigma_{t}\right) \mathrm{d} t\right)\right)<\infty$ for some $\gamma>0$.

Indeed, consider

$$
\mathbf{E}\left(\int_{t}^{\infty} h\left(\sigma_{u}\right) \mathrm{d} u \mid \mathcal{F}_{t}\right)=\mathbf{E}\left(\int_{t}^{\infty} h\left(\sigma_{u}\right) \mathrm{d} u \mid \sigma_{t}\right)=\mathbf{E}_{\sigma_{t}}\left(\int_{0}^{\infty} h\left(\sigma_{u}\right) \mathrm{d} u\right) \leqslant \mathbf{E}\left(\int_{0}^{\infty} h\left(\sigma_{u}\right) \mathrm{d} u\right),
$$

because $\sigma$ is increasing and $h$ is decreasing. Therefore, the condition (17) with

$$
A_{t}:=\int_{0}^{\infty} h\left(\sigma_{u}\right) \mathrm{d} u
$$

holds implying the existence of some exponential moments.

(3) A version of Lemma 5.2 can be found already in Meyer [20], Chapter VII, Section 6: Quelques résultats sur l'énergie; see, in particular, the inequality (59.2), p. 182

$$
\mathbf{E}\left(A_{\infty}^{n}\right) \leqslant n ! C^{n},
$$

where $\left\{A_{t}\right\}$ denotes an increasing integrable predictable process (called "natural" by Meyer in 1966), whose potential is bounded by $C$. The special case with $n=2$ appears as a Corollary (formula (24.1)) to Théorème 23 which gives an integral expression for $\mathbf{E}\left(A_{\infty}^{2}\right)$.

(4) Lemma 5.2 plays also an important rôle in the studies of BMO-martingales, see e.g. Kazamaki and Sekiguchi [18], Emery [9] and Kazamaki [17], Theorem 2.2, p. 29. We also refer to Engelbert and Schmidt [11] where (17) is used to derive more explicit sufficient conditions for an exponential local martingale associated with a diffusion martingale to be a martingale. In particular, for a Brownian motion $B$ and a fixed $t>0$, it follows from Corollary (3.16) in [11] that

$$
\int_{0}^{t} f\left(B_{S}\right) \mathrm{d} s
$$

has some exponential moments if $f$ is locally integrable and bounded at infinity.

\section{Exponential moments - class $\mathcal{K}_{3}$}

Proposition 6.1. $\left(\mathcal{K}_{3}\right)$ Assume that the function $f$ satisfies $(\mathrm{A})$, is integrable at $+\infty$, and

$$
f(x)=\mathrm{O}(1)_{x \rightarrow-\infty} .
$$

Then there exists $\gamma>0$ such that for all $x$

$$
\mathbf{E}_{x}\left(\exp \left(\gamma I_{\infty}(f)\right)\right)<\infty .
$$

In particular, if $f(x) \leqslant C_{N}$ for all $x \leqslant N$ then (20) holds for

$$
\gamma<\min \left\{\frac{\mu}{2 \int_{N}^{\infty} f(x) \mathrm{d} x}, \frac{\mu^{2}}{4 C_{N}}\right\} .
$$


Proof. By (19), there exists $N$ such that $f$ is bounded in $(-\infty, N)$. We prove first that (20) holds for a function $f$ satisfying the stated assumptions and for which there exists $x^{*}$ such that $\{f>0\} \subset\left(-\infty, x^{*}\right)$ and $f$ is bounded in $\left(-\infty, x^{*}\right)$ by a constant $C$. Let

$$
\lambda_{x^{*}}:=\sup \left\{t: B_{t}^{(\mu)}=x^{*}\right\}
$$

Then

$$
\int_{0}^{\infty} f\left(B_{t}^{(\mu)}\right) \mathrm{d} t=\int_{0}^{\lambda_{x^{*}}} f\left(B_{t}^{(\mu)}\right) \mathrm{d} t \leqslant C \lambda_{x^{*}} .
$$

The $\mathbf{P}_{x}$-distribution of $\lambda_{x^{*}}$ with $x \leqslant x^{*}$ has the Laplace transform (see Pitman and Yor [21] and Borodin and Salminen [2], p. 27)

$$
\mathbf{E}_{x}\left(\exp \left(-\alpha \lambda_{x^{*}}\right)\right)=\frac{G_{\alpha}\left(x, x^{*}\right)}{G_{0}\left(x^{*}, x^{*}\right)}
$$

where the Green kernel $G_{\alpha}, \alpha \geqslant 0$, is given for $x \leqslant x^{*}$ by (cf. Borodin and Salminen [2], A1.14, p. 127, see also (7))

$$
G_{\alpha}\left(x, x^{*}\right):=\frac{1}{2 \sqrt{2 \alpha+\mu^{2}}} \mathrm{e}^{-\left(\sqrt{2 \alpha+\mu^{2}}+\mu\right) x^{*}} \mathrm{e}^{\left(\sqrt{2 \alpha+\mu^{2}}-\mu\right) x} .
$$

Consequently, studying (23), it is seen that for $\gamma<\mu^{2} / 2$

$$
\mathbf{E}_{x}\left(\exp \left(\gamma \lambda_{x^{*}}\right)\right)<\infty
$$

and this implies, using (22), for $\gamma<\mu^{2} /(2 C)$

$$
\mathbf{E}_{x}\left(\exp \left(\gamma I_{\infty}(f)\right)\right)<\infty
$$

Consider next a function $f$ for which the made assumptions hold and for which there exists $x^{*}$ such that $\{f>0\} \subset$ $\left(x^{*}, \infty\right)$. Then $f \in \mathcal{K}_{4}$ and we have (20) by Proposition 5.1(ii). Let now $f$ be an arbitrary function satisfying the assumptions and introduce

$$
f_{1}(x):=f(x) \mathbf{1}_{(-\infty, 0)}(x), \quad \text { and } \quad f_{2}(x):=f(x) \mathbf{1}_{(0,+\infty)}(x) .
$$

Then, as explained above, $f_{2} \in \mathcal{K}_{4} \subset \mathcal{K}_{3}$, and also $f_{1} \in \mathcal{K}_{3}$ because, without loss of generality, we may assume that $f_{1}$ is bounded on $(-\infty, 0)$. The Cauchy-Schwarz inequality implies that $f \in \mathcal{K}_{3}$. The bound given in (21) is obtained with a similar reasoning as above (using $N$ instead of 0 ) combined with the bound in Proposition 5.1, details are left to the reader.

Remark 6.2. Notice that if $f$ is bounded then, writing

$$
f(x)=f(x) \mathbf{1}_{(-\infty, N)}(x)+f(x) \mathbf{1}_{(N,+\infty)}(x)
$$

with $N$ large as in the proof above but using the $\mathbf{L}^{p}$-inequality instead, it follows

$$
\mathbf{E}_{x}\left(\exp \left(\gamma \int_{0}^{\infty} f\left(B_{t}^{(\mu)}\right) \mathrm{d} t\right)\right)<\infty,
$$

for $\gamma<\mu^{2} /(2 C)$ where $C:=\sup \{f(x): x \in \mathbf{R}\}$.

Example 6.3. Consider the functional in (2), that is, $f(x)=(1+a \exp (x))^{-2}$. Then $f \in \mathcal{K}_{3}$. Because $f$ is bounded by 1 , the functional $I_{\infty}(f)$ has exponential moments for $\gamma<\mu^{2} / 2$. 


\section{Note added in proof}

The Laplace transform of the functional in (2) has now been computed in: A.N. Borodin, P. Salminen, On some exponential integral functionals of $\operatorname{BM}(\mu)$ and BES(3), Zap. Nauchn. Sem. POMI 311 (2004) 57-78.

\section{Acknowledgements}

We thank Hans-Jürgen Engelbert for sending us the paper [13] and also for comments on our work.

\section{References}

[1] J. Bertoin, Subordinators: Examples and applications, in: J. Bertoin, F. Martinelli, Y. Peres (Eds.), École d'eté de Probabilités de SaintFlour XXVII-1997, in: Lecture Notes in Math., vol. 1717, Springer, Berlin, 1999, pp. 1-91.

[2] A.N. Borodin, P. Salminen, Handbook of Brownian Motion - Facts and Formulae, second ed., Birkhäuser, Basel, 2002.

[3] A.S. Cherny, Convergence of some integrals associated with Bessel processes, Theoret. Probab. Appl. 45 (2000) $251-267$.

[4] K.L. Chung, Z. Zhao, From Brownian Motion to Schrödinger's Equation, Springer-Verlag, Berlin, 1995.

[5] M. Csörgö, L. Horváth, Q.M. Shao, Convergence of integrals of uniform empirical and quantile processes, Stochastic Process. Appl. 45 (1993) 278-294.

[6] C. Dellacherie, P.A. Meyer, Probabilités et Potentiel, vols. I-V, Hermann, Paris, 1975-1992.

[7] D. Dufresne, The distribution of a perpetuity, with applications to risk theory and pension funding, Scand. Actuar. J. 1-2 (1990) 39-79.

[8] R. Durrett, Brownian Motion and Martingales in Analysis, Wadsworth, Belmont, CA, 1984.

[9] M. Emery, Une définition faible de BMO, Ann. Inst. H. Poincare 21 (1985) 59-72.

[10] H.J. Engelbert, W. Schmidt, On the behaviour of certain functionals of the Wiener process and applications to stochastic differential equations, in: Stochastic Differential Systems. Proc. 3rd IFIP-WG 7/1 Working Conf., in: Lecture Notes in Control and Inform. Sci., vol. 36, Springer-Verlag, Berlin, 1981, pp. 47-55.

[11] H.J. Engelbert, W. Schmidt, On exponential local martingales connected with diffusion processes, Math. Nachr. 119 (1984) $97-115$.

[12] H.J. Engelbert, W. Schmidt, On the behaviour of certain Bessel functionals. An application to a class of stochastic differential equations, Math. Nachr. 131 (1987) 219-234.

[13] H.J. Engelbert, T. Senf, On functionals of Wiener process with drift and exponential local martingales, in: M. Dozzi, H.J. Engelbert, D. Nualart (Eds.), Stochastic Processes and Related Topics, Proc. Wintersch. Stochastic Processes, Optim. Control, Georgenthal/Ger. 1990, in: Math. Res., vol. 61, Academic-Verlag, Berlin, 1991, pp. 45-58.

[14] P. Fitzsimmons, J. Pitman, Kac's moment formula and the Feynman-Kac formula for additive functionals of a Markov process, Stochastic Process. Appl. 79 (1999) 117-134.

[15] T. Jeulin, Semimartingales et grossissement d'une filtration, Lecture Notes in Math., vol. 833, Springer-Verlag, Berlin, 1980.

[16] T. Jeulin, Sur la convergence absolue de certaines intégrales, in: J. Azéma, M. Yor (Eds.), Séminaire de Probabilités XVI, in: Lecture Notes in Math., vol. 920, Springer, Berlin, 1982, pp. 248-256.

[17] N. Kazamaki, Continuous Exponential Martigales and BMO, Lecture Notes in Math., vol. 1579, Springer-Verlag, Berlin, 1994.

[18] N. Kazamaki, T. Sekiguchi, On the transformation of some classes of martingales by a change of law, Tôhoku Math. J. 31 (1979) $261-279$.

[19] R. Khas'minskii, On positive solutions of the equation $A u+V u=0$, Theoret. Probab. Appl. 4 (1959) 309-318.

[20] P.A. Meyer, Probabilités et potential, Hermann (Editions Scientifiques), Paris, 1966.

[21] J. Pitman, M. Yor, Bessel processes and infinitely divisible laws, in : D. Williams (Ed.), Stochastic Integrals, in : Lecture Notes in Math., vol. 851, Springer-Verlag, Berlin, 1981, pp. 285-370.

[22] J. Pitman, M. Yor, A decomposition of Bessel bridges, Z. Wahrsch. Verw. Gebiete 59 (1982) 425-457.

[23] J. Pitman, M. Yor, Some divergent integrals of Brownian motion, in : D. Kendall (Ed.), Analytic and Geometric Stochastics, Adv. Appl. Probab. (Supplement) (1986) 109-116.

[24] P. Salminen, M. Yor, Perpetual integral functionals as hitting times, Elec. J. Prob., in press.

[25] L.A. Shepp, Radon-Nikodym derivatives of Gaussian measures, Ann. Math. Statist. 37 (1966) 321-354.

[26] B. Simon, Functional Integration and Quantum Physics, Academic Press, New York, 1979.

[27] W. Stummer, K.-T. Sturm, On exponentials of additive functionals of Markov processes, Stochastic Process. Appl. 85 (2000) $45-60$.

[28] X.-X. Xue, A zero-one law for integral functionals of the Bessel process, in : J. Azéma, P.A. Meyer, M. Yor (Eds.), Séminaire de Probabilités XXIV, in : Lecture Notes in Math., vol. 1426, Springer, Berlin, 1990, pp. 137-153.

[29] M. Yor, Sur certaines fonctionnelles exponentielles du mouvement brownien réel, J. Appl. Probab. 29 (1992) $202-208$.

[30] M. Yor, Exponential Functionals of Brownian Motion and Related Processes, Springer Finance, Springer-Verlag, Berlin, 2001. 